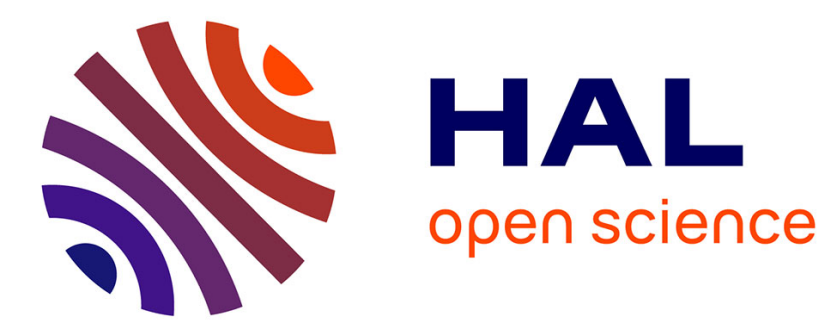

\title{
Cherenkov radiation from relativistic heavy ions taking account of their slowing down in radiator
}

\author{
V.R. Altapova, O.V. Bogdanov, Yu.L. Pivovarov
}

\section{To cite this version:}

V.R. Altapova, O.V. Bogdanov, Yu.L. Pivovarov. Cherenkov radiation from relativistic heavy ions taking account of their slowing down in radiator. The Seventh International Symposium on Swift Heavy Ions in Matter, Jun 2008, Lyon, France. hal-00256951

\section{HAL Id: hal-00256951 https://hal.science/hal-00256951}

Submitted on 12 Dec 2008

HAL is a multi-disciplinary open access archive for the deposit and dissemination of scientific research documents, whether they are published or not. The documents may come from teaching and research institutions in France or abroad, or from public or private research centers.
L'archive ouverte pluridisciplinaire HAL, est destinée au dépôt et à la diffusion de documents scientifiques de niveau recherche, publiés ou non, émanant des établissements d'enseignement et de recherche français ou étrangers, des laboratoires publics ou privés. 


\title{
Cherenkov radiation from relativistic heavy ions taking account of their slowing down in radiator
}

\author{
V. R. Altapova ${ }^{a, b}$, O.V. Bogdanov ${ }^{a, b}$, Yu. L. Pivovarov ${ }^{a, b, *}$ \\ ${ }^{a}$ Tomsk Polytechnic University, Lenin Ave. 30, 634050 Tomsk, Russia \\ ${ }^{b}$ Nuclear Physics Institute, Lenin Ave. 2-A, 634050 Tomsk, Russia
}

\begin{abstract}
Combining the SRIM'06 computer code and the formula for spectral-angular distribution of radiation from a particle moving in a medium in arbitrary trajectory, we studied numerically the structure of angular distributions of Cherenkov radiation from moderately relativistic heavy ions (RHI) taking into account the decrease of the ion velocity due to stopping in the radiator. The results obtained clearly show that the width and the fine structure of the Cherenkov radiation in the vicinity of the Cherenkov cone depend strongly on several factors, e.g. the mean and total energy losses, the radiator thickness, the square of the ion charge, the emission wavelength and the refractive index of the radiator material. This might be useful for experiments using RICH detectors for relativistic heavy ions.
\end{abstract}

PACS: $61.85 .+p, 78.70 .-\mathrm{g}, 41.60 .-\mathrm{m}$

\section{Introduction}

The Tamm-Frank's theory [1] describes the Cherenkov radiation (ChR) of a particle that moves rectilinearly with a constant velocity $v$ in a medium of refractive index $n$ larger than $v / c$ ( $c$ is the speed of light in vacuum). This theory neglects both the bending of the particle trajectory (due to multiple scattering) and the decrease of its velocity (due to ionization energy loss).

Dedrick [2] was the first to take into account the influence of the particle-radiator interaction on the angular density of the particle's Cherenkov radiation. He examined the influence of multiple scattering, while ignoring the slowing-down effect.

Kuzmin and Tarasov [3] considered another limiting case where the multiple scattering effect is insignificant, and it is the slowing-down effect that plays a key role in forming the angular distribution of Cherenkov radiation. It occurs when heavy relativistic heavy ions $(Z, A>>1)$ cross thin radiators. They ignored the fluctuations of ionization energy loss and the trajectory bending effect due to multiple scattering and studied the angular distribution of the Cherenkov radiation emitted by an ion moving rectilinealy but at a decreasing velocity. This assumption allowed them to obtain the formula for calculating the angular density of Cherenkov radiation when taking into account the slowing down in the thin radiator. The thin radiator approximation was used in [3] to obtain the simple expression for the velocity decreasing with the penetration depth $x$ due to ionization energy loss, in the form: $\frac{1}{v(x)} \approx \frac{1}{v_{0}}-\frac{1}{v_{0}^{2}}\left(\frac{d v(x)}{d x}\right)_{x=0} \cdot x$, where $v_{0}$ is the initial velocity of the ion. Since the apertura of the Cherenkov emission cone is fixed by the particle velocity, $\cos \vartheta_{C}=c / n v=1 / \beta n$, it is clear that one may expect an additional broadening $\Delta \vartheta$ of the angular distribution, the value of

\footnotetext{
* Corresponding author

E-mail address: pivovarov@tpu.ru (Yu. L. Pivovarov)
} 
which is determined approximately by the initial and final velocities of the particle. In the expression above, the velocity gradient is expressed through the stopping power $d E / d x$ of the radiator:

$$
\frac{d v}{d x}=\frac{d E}{d x} \cdot \frac{d v}{d E}=\frac{1}{p \gamma^{2}} \cdot \frac{d E}{d x} ; p=v E / c^{2}
$$

Here, $p, E, \gamma$ are the momentum, the energy and the relativistic factor of the ion. Thus, $\Delta \vartheta$ becomes proportional to the stopping power in radiator and to the radiator thickness $L$. Within $\Delta \vartheta$, the authors of Ref. [3] predict the diffraction-like structure in the angular distribution, with a diffraction parameter (the distance between maxima) depending on the emission wavelength, the initial velocity and the stopping power $d E / d x$ of the radiator. Of course, the analytical formulae obtained in Ref.[3] contain (implicitly) the dependence of the $\mathrm{ChR}$ angular distribution on the square of the ion charge, the radiator thickness $\mathrm{L}$ (until the radiator is thin) and the emission wavelength. However, numerical calculations presented in Ref.[3] are performed only for the fixed beam energy 1000 $\mathrm{MeV} / \mathrm{u}$, only for the fixed emission wavelength $546.1 \mathrm{~nm}$ and only for three types of ions: $\mathrm{Ne}, \mathrm{Fe}$, $\mathrm{Au}$.

In order to avoid the approximations made in [3] for the thin radiator, here we developed a new approach which uses the popular computer code SRIM'06 [5] to calculate the velocity of an ion depending on its penetration depth in the radiator, and we subsequently substituted it into the formula of classical electrodynamics for the spectral-angular distribution of radiation from a particle moving in a medium along a trajectory $\boldsymbol{r}(t)$ with taking into account the slowing-down due to ionization energy loss in the radiator. The new suggested method allowed us to investigate in more detail dependences of the $\mathrm{ChR}$ angular distributions on the square of the ion charge, the radiator thickness, the emission wavelength and the refractive index of the radiator material.

\section{Theory and calculation scheme}

The general expression for the intensity of the radiation from RHI with a charge $Z e$ penetrating through a non-magnetic dielectric medium characterized by the dielectric function $\varepsilon=\varepsilon(\omega)$ may be written in the form (see, e.g. [4]):

$$
\frac{d I}{d \omega d \Omega}=\frac{Z^{2} e^{2} \omega^{2}}{4 \pi^{2} c^{3}} \sqrt{\varepsilon} \int_{-\infty}^{+\infty} \mathbf{n} \times[\mathbf{n} \times \mathbf{v}] \cdot \exp \left\{i \omega\left[t-\frac{\sqrt{\varepsilon} \cdot \mathbf{n} \cdot \mathbf{r}(t)}{c}\right]\right\} d t \mid
$$

In the case of rectilinear motion $r(t)=v \cdot t$, therefore

$$
\frac{d I}{d \omega d \Omega}=\frac{Z^{2} e^{2}}{c^{3}} \sqrt{\varepsilon}[\boldsymbol{n} \times \boldsymbol{v}]^{2}\left|\frac{\omega}{2 \pi} \int_{-\infty}^{+\infty} \exp \left\{i \omega t\left[1-\frac{\sqrt{\varepsilon}}{c} \boldsymbol{n} \cdot \boldsymbol{v}\right]\right\} d t\right| \text {. }
$$

The integral in the above expression is the Dirac $\delta$-function, that means

$$
\frac{d I}{d \omega d \Omega}=\frac{Z^{2} e^{2}}{c} \sqrt{\varepsilon} \beta^{2} \sin ^{2} \vartheta \mid \delta(1-\sqrt{\varepsilon} \beta \cos \vartheta)^{2} \text {. }
$$

Here, $\vartheta$ is the angle between the emission direction and the velocity vector $v$. The appearance of $\delta$ - function in expression (3) is due to infinite penetration time with a constant velocity and leads to infinite spectral density of the radiation emitted at the Cherenkov angle. If one takes into account the finite time $2 T$ of RHI penetration through the radiator, then the divergence disappears:

$$
\frac{\omega}{2 \pi} \int_{-\infty}^{+\infty} \exp \left\{i \omega t\left[1-\frac{\sqrt{\varepsilon}}{c} \boldsymbol{n} \cdot \boldsymbol{v}\right]\right\} d t=\frac{\omega T}{\pi} \cdot \frac{\sin [\omega T(1-\sqrt{\varepsilon} \beta \cos \vartheta)]}{\omega T(1-\sqrt{\varepsilon} \beta \cos \vartheta)} \text {. }
$$

Thus, the spectral-angular distribution of the Cherenkov radiation (Tamm-Frank distribution) from $\mathrm{RHI}$ in the finite-size radiator is defined by following equations: 


$$
\begin{aligned}
& \frac{d I}{d \omega d \Omega}=\omega \cdot L \cdot\left(\frac{Z e \cdot \sin \vartheta}{c}\right)^{2} \cdot f_{T F}(\theta, \omega) \\
& f_{T F}(\theta, \omega)=\frac{1}{\Delta \vartheta_{T F}} \cdot\left(\frac{\sin x}{x}\right)^{2} ; x=\frac{\pi}{\Delta \vartheta_{T F}}\left(\cos \vartheta-\frac{1}{\beta n}\right) \\
& \Delta \vartheta_{T F}=\frac{\lambda}{n L} ; \quad \lambda=\frac{2 \pi c}{\omega}
\end{aligned}
$$

The width $\Delta \vartheta_{T F}=\lambda / n L$ of the Tamm-Frank distribution (concentrated in the vicinity of the Cherenkov cone) is inversely proportional to the radiator thickness $L$. Therefore, at large $L$ there appears a very sharp maximum at the Cherenkov angle $\vartheta=\vartheta_{C}$ defined by $\cos \vartheta_{C}=1 / \beta n$.

In the case of RHI penetrating through a radiator, we may assume that the multiple scattering effect is negligible, i.e. the direction of RHI velocity $\mathbf{v} / v=$ const but its magnitude decreases due to ionization energy loss, i.e. depends on penetration depth $x, v=v(x)$. As in [3], we can replace the integration over time in Eq.(2) by the integration over the trajectory, by making use of the substitution

$$
t(x)=\int_{0}^{x} \frac{d x^{\prime}}{v\left(x^{\prime}\right)}
$$

After some algebra we arrive at the following expression for the spectral-angular distribution of $\mathrm{ChR}$ from RHI, taking into account the stopping in the radiator:

$$
\begin{aligned}
& \frac{d I}{d \omega d \Omega}=\omega \cdot L \cdot\left(\frac{Z e \cdot \sin \vartheta}{c}\right)^{2} \cdot f(\theta, \omega) \\
& f(\theta, \omega)=\frac{1}{L^{2}}\left|\int_{0}^{L} \exp \left\{i \omega\left[t(x)-\frac{\sqrt{\varepsilon} \cdot x \cdot \cos \theta}{c}\right]\right\} d x\right|^{2} ; t(x)=\int_{0}^{x} \frac{d x^{\prime}}{v\left(x^{\prime}\right)}
\end{aligned}
$$

According Eq.(8), the angular distribution of Cherenkov radiation in the vicinity of the Cherenkov angle is characterized by the new distribution function $f(\theta, \omega)$. This function was calculated analytically in Ref. [3] for the case of a thin radiator. The key step in our calculation is the use of the SRIM'06 package [5] for calculations of stopping and its dependence on the penetration depth velocity $v(x)$ of RHI. Then the calculated value of $v(x)$ is used in the equations (8) for $f(\theta, \omega)$. An example of these calculations of $v(x)$ is presented in Fig. 1.
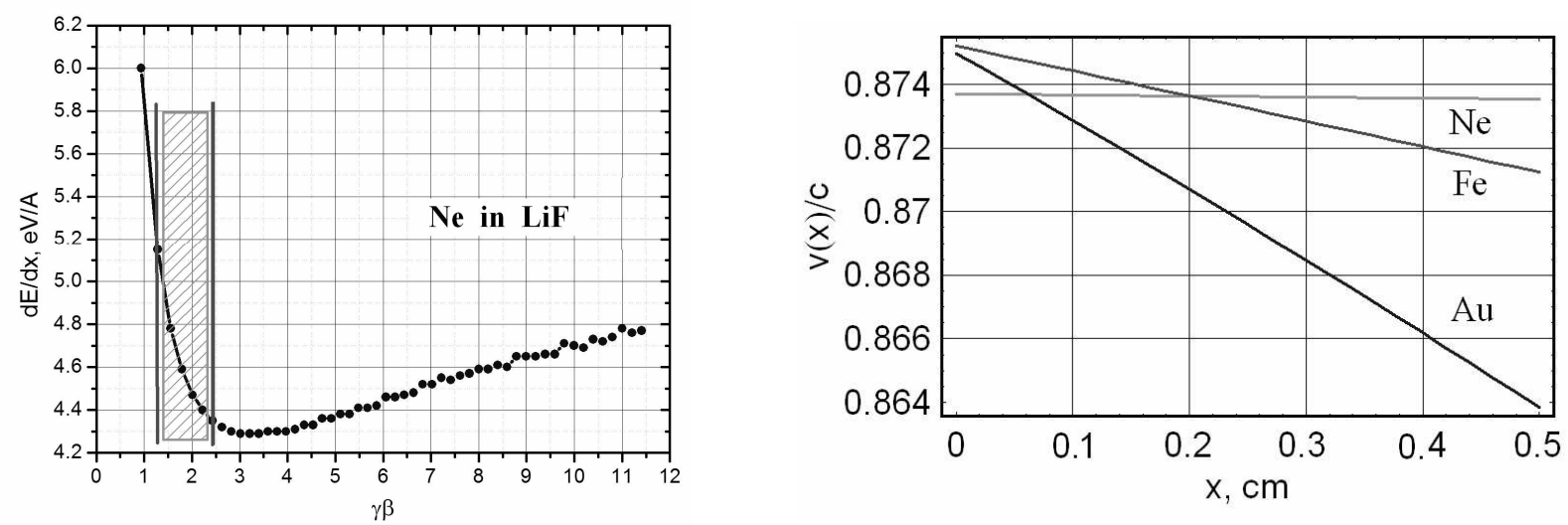

Figure 1. Stopping power (left) for $\mathrm{Ne}$ in a $\mathrm{LiF}$ radiator and the velocity as a function of the penetration depth calculated by SRIM'O6 (right) for $\mathrm{Ne}, \mathrm{Fe}, \mathrm{Au}$ ions in $\mathrm{LiF}$ (the incident ion beam energy is $1000 \mathrm{MeV} / \mathrm{u}$ ). The left vertical line of the dashed zone denotes the threshold value of $\gamma \beta$ for appearance of the $\mathrm{ChR}$ while the right vertical line denotes $\gamma \beta$ value for $\mathrm{E}=1000 \mathrm{MeV} / \mathrm{u}$. 


\section{Influence of RHI stopping on angular distributions of ChR: results of calculations}

Based on formula (8) and the SRIM calculations of $v(x)$, we performed three sets of calculations, in order to study the dependence of the ChR angular distribution from $\mathrm{RHI}$ on the charge $Z e$, the radiator thickness $L$ and the emission wavelength $\lambda$.

\section{1. $\mathbf{Z}^{2}$ Dependence of $\mathrm{ChR}$ angular distributions from $\mathrm{RHI}$}

Since the RHI stopping in the radiator is proportional to square of the ion charge, $(Z e)^{2}$ for fixed thickness of the radiator and emission wavelength we may expect a significant change of both the velocity and the distribution function $f(\theta, \omega)$ depending on $\mathrm{Z}$. The results of numerical calculations are presented in Fig. 2 and confirm this suggestion.

Figures 2 a-d clearly show that stopping of RHI in the radiator results in the drastic change of angular distribution of RHI compared to the Tamm-Frank distribution. The latter almost corresponds to the distribution shown in the Fig. $2 \mathrm{~d}$, since the stopping of light Be ions $(Z=4)$ at the given penetration depth is still small. In contrast, the stopping for heavy $U$ ions and corresponding change of the velocity becomes significant, which leads to a remarkable broadening of the angular distribution and its diffraction-like structure, similar to the spectra predicted earlier in [3]. One should mention that the Tamm-Frank formula (5-6) predicts only the $Z^{2}$ dependence of the total intensity of ChR, but not its angular dependence on the particle (ion) charge. The analysis of the $Z^{2}$ dependence of other kinds of radiation from RHI in crystal targets was performed earlier in [6].
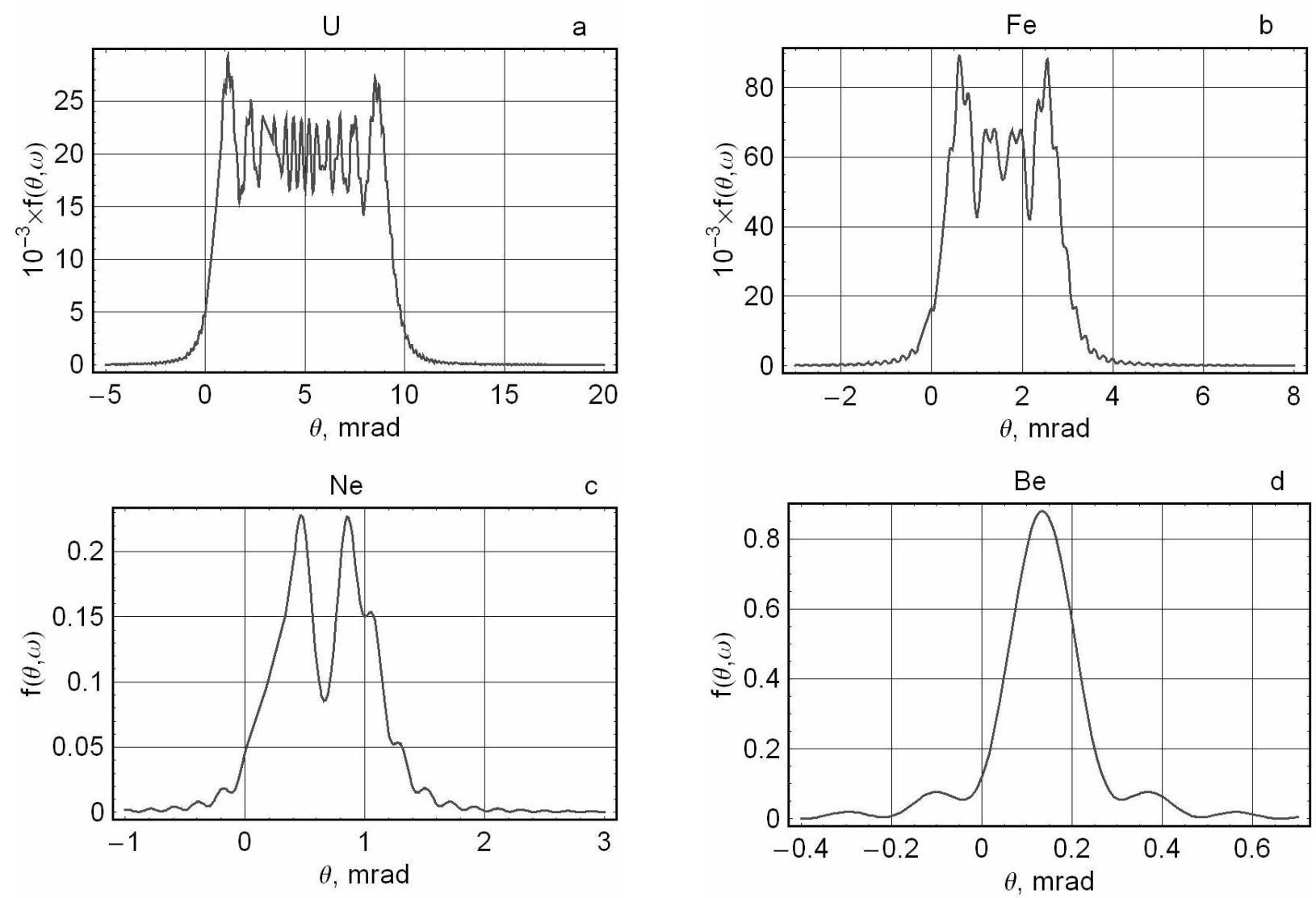

Figure 2. ChR angular distribution in the vicinity of the Cherenkov cone for $U(Z=92), F e(Z=26)$, $\mathrm{Ne}(Z=10)$ and $\mathrm{Be}(Z=4)$. Other parameters: radiator material $-\mathrm{LiF}$; radiator length $L=0.25 \mathrm{~cm}$; 
emission wavelength $\lambda=390 \mathrm{~nm}$; index of refraction $n=\sqrt{\varepsilon}=1.338$ [6]; initial ion beam energy $1000 \mathrm{MeV} / \mathrm{u}$. Here, $\vartheta=0$ corresponds to Cherenkov angle $\vartheta=\vartheta_{C}, \cos \vartheta_{C}=1 / \beta n$ and positive $\vartheta$ values in fact denote emission angles smaller than $\vartheta_{C}$.

\section{2. $\mathrm{L}$ - Dependence of $\mathrm{ChR}$ angular distributions from RHI}

Since the RHI stopping in the radiator is proportional to the radiator thickness $L$, then for fixed values of the ion charge and of the emission wavelength we may expect a greater change of velocity and distribution function $f(\theta, \omega)$ with the increase of the radiator thickness $L$. The results of the numerical calculations are presented in Fig. 3 and confirm this suggestion.
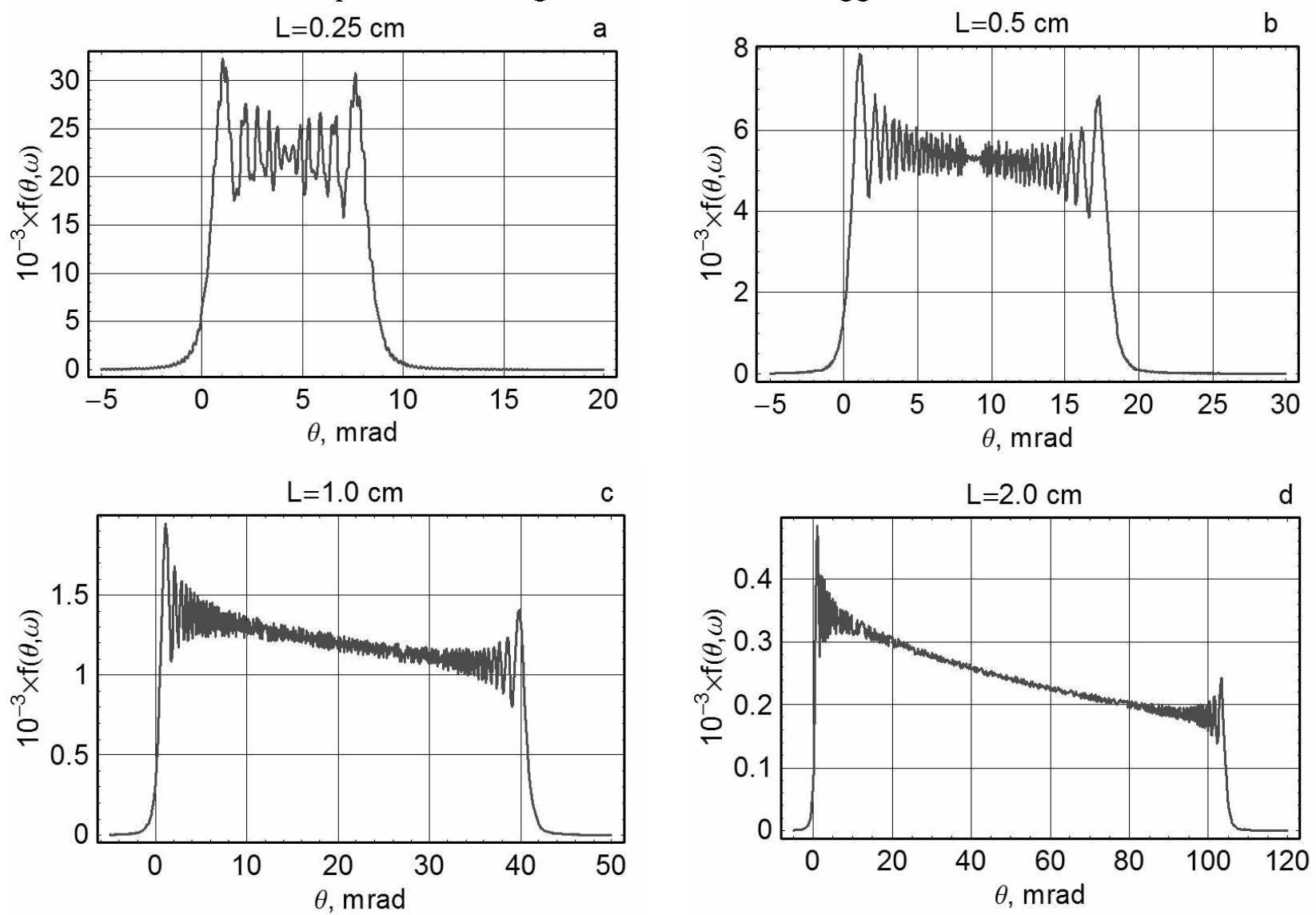

Figure 3. Broadening of the $\mathrm{ChR}$ angular distribution from $\mathrm{Au}$ ion $(Z=79)$ in the vicinity of the Cherenkov cone with the increase of the radiator length $L$ from $L=0.25 \mathrm{~cm}$ up to $L=2.0 \mathrm{~cm}$. Other parameters: radiator material $-\mathrm{LiF}$; emission wavelength $\lambda=390 \mathrm{~nm}$; index of refraction $n=\sqrt{\varepsilon}=1.40$ [6]; initial ion beam energy $1000 \mathrm{MeV} / \mathrm{u}$. As in Fig. $2, \vartheta=0$ corresponds to Cherenkov angle $\vartheta=\vartheta_{C}, \cos \vartheta_{C}=1 / \beta n$ and positive $\vartheta$ values in fact denote emission angles smaller than $\vartheta_{C}$.

Figures 3 a-d clearly show that the stopping of RHI in the radiator again results in a drastic change of the angular distribution of RHI compared to the Tamm-Frank distribution of ChR for the finite-size radiator. The latter is almost similar to the distribution shown in the Fig. 2a, but becomes narrower with increase of the radiator thickness $L$, since its width is inversely proportional to $L$ (see, Eq.(6)). In contrary, the stopping of RHI in the radiator leads to a remarkable broadening of the angular dis- 
tribution with the increase of the radiator length $L$. In addition, there appears again a diffraction-like structure of angular distributions, which is washed out at large $L$ values.

\section{3. $\lambda$ - Dependence of ChR angular distributions from RHI}

As is well known since Tamm-Frank [1], the wavelength $\lambda$ (or frequency $\omega$ ) of emitted Cherenkov radiation determines the direction of Cherenkov emission, i.e. defines the Cherenkov angle $\vartheta_{C}$ $\cos \vartheta_{C}=1 / \beta n$. The wavelength $\lambda$ also enters Eq.(3) which determines the width of the Tamm-Frank distribution in the finite-size radiator, so that the width is proportional to $\lambda$.

Besides, according Equation (8), the wavelength (or frequency) of emitted Cherenkov radiation influences the angular distribution through its presence in the exponential multiplier in the integrand of Eq.(8).

The results of numerical calculations are presented in Fig. 4 and confirm this suggestion.
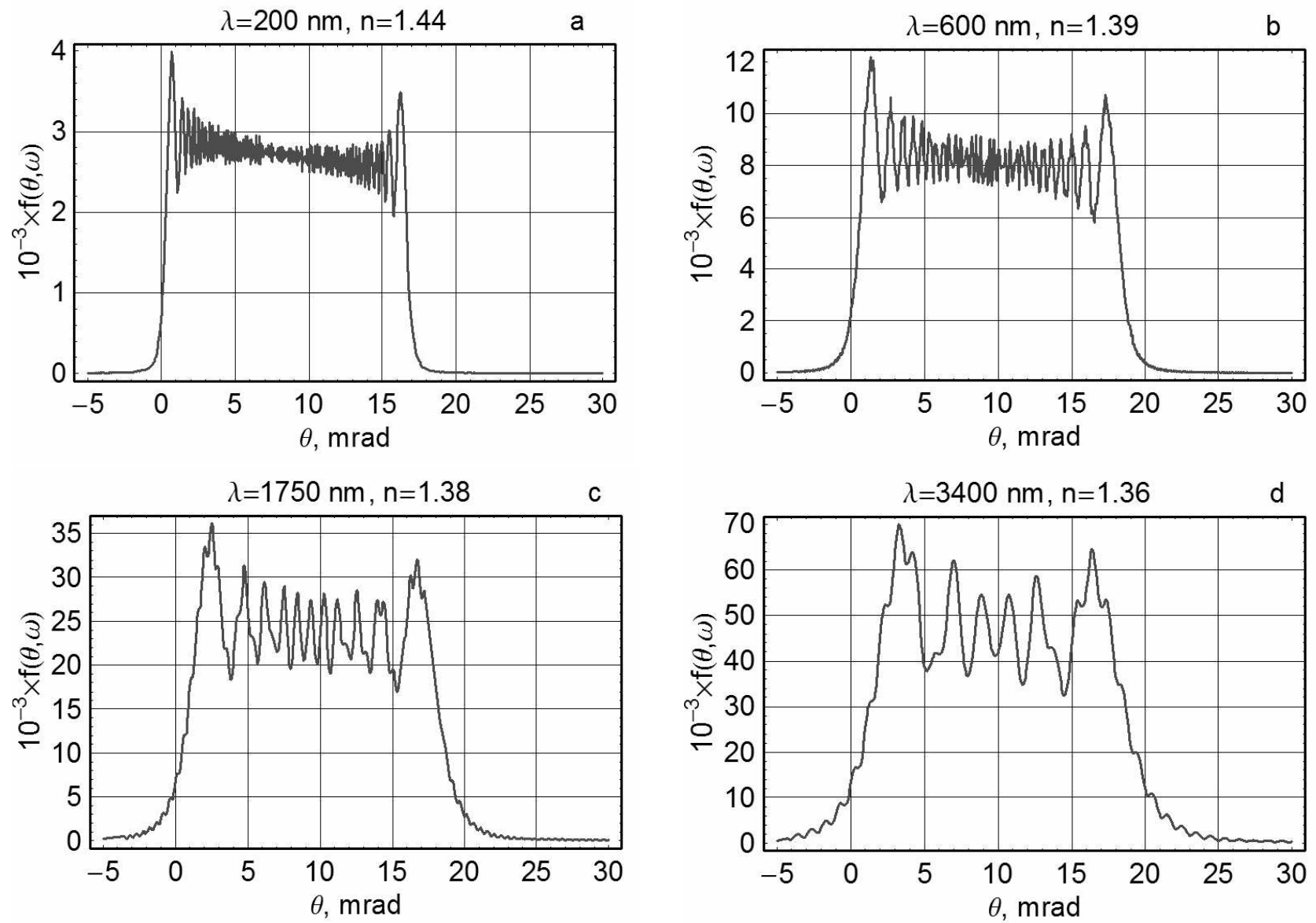

Figure 4. Change of the $\mathrm{ChR}$ angular distribution from $\mathrm{Au}$ ion $(Z=79)$ in the vicinity of the Cherenkov cone with the increase of the emission length $\lambda$ from $\lambda=200 \mathrm{~nm}$ up to $\lambda=3400 \mathrm{~nm}$. Other parameters are: radiator material - LiF; radiator thickness $L=0.5 \mathrm{~cm}$; index of refraction $n=\sqrt{\varepsilon}$ depends on $\lambda$ according [6]; initial ion beam energy $1000 \mathrm{MeV} / \mathrm{u}$. As in Fig. 2, $\vartheta=0$ corresponds to Cherenkov angle $\vartheta=\vartheta_{C}, \cos \vartheta_{C}=1 / \beta n$ and positive $\vartheta$ values in fact denote emission angles smaller than $\vartheta_{C}$.

Figures 4 a-d clearly show that the influence of stopping of RHI in the radiator of the fixed thickness manifests itself more with increase of emission wavelength. Indeed, while the width of distribution shown in Fig 2a-d is almost the same, for longer wavelengths the diffraction-like structure becomes 
more prominent. This has a very clear physical meaning reflecting the breakdown of the coherence condition for emission (which depends on $\lambda$ ) along RHI trajectory due to slowing down.

\section{Conclusions}

The direct implementation of SRIM'06 into calculations of Cherenkov radiation angular distribution from moderately relativistic heavy ions, taking into account the stopping in the radiator, has been performed. The results of calculations show that: the angular distribution of the Cherenkov radiation in the vicinity of the Cherenkov cone has a complicated fine structure and depends strongly on

- Square of the ion charge $Z^{2}$

- Radiator thickness $L$

- Emission wave length $\lambda$ and corresponding index of refraction $n(\lambda)$

The experimental studies of predicted effects are possible at existing and future relativistic heavy ion accelerators, for example using RICH detectors [8]. The possible applications of $Z^{2}$ dependence of angular distribution, such as measurements of particle charge, incident energy, even mean energy loss in the radiator are clearly seen and will be discussed in details in the next paper.

These experiments could be e.g. complementary to experiments on parametric X-radiation (PXR, or quasi-Cherenkov radiation) from relativistic nuclei in crystals [9] or proposed ones on resonant coherent excitation of relativistic heavy ions at FAIR [10].

The authors thank the Tomsk Polytechnic University and Nuclear Physics Institute, Tomsk Polytechnic University for financial support.

\section{References:}

[1] Tamm I.E., Frank I.M. Doklady AN SSSR, 1937, 14, p. 107.

[2] Dedrick K. G. Phys. Rev., 1952, 87, p. 891.

[3] Kuzmin E.S, Tarasov A.V. JINR Rapid Communications 4[61]-93 (1993), p.64.

[4] Jackson J.D. Classical Electrodynamics 2nd version, John \& Wiley Sons, New York, 1975

[5] URL http://www. srim.org

[6] Springer Handbook of Condensed Matter and Materials Data, Berlin Heidelberg, 2005.

[7] Pivovarov Yu.L., Kunashenko Yu.P., Vorobiev S.A. Radiation Effects, 1986, V.100, P.51.

[8] RICH detectors: Grupen C. Particle Detectors, Cambridge University Press, 1996; CERN Courier, V 47, N 6, 2007; GSI-Nachrichten, 3/97

[9] Adischev Yu.N.et al, V.N. Nucl. Instr. Meth. Phys. Res.B 252 (2006) p.111-117

[10] Pivovarov Yu.L., Geissel H., Scheidenberger C. Nucl. Instr. Meth. Phys. Res.B 256 (2007) p. 109-113 